\title{
Significance of $\beta$-Crosslaps and the tumour markers in diseases of the prostate gland
}

\author{
Lukas J. Becker* and Gerhard M. Oremek \\ Departments of Laboratory Medicine - Internal Medicine, Hospital of the Johann Wolfgang Goethe University, D-60590 Frankfurt/Main, Germany
}

\begin{abstract}
Objective of this study was to assess the validity of $\beta$-Crosslap concerning prostate carcinoma and to evaluate its benefit in the early detection, the diagnosis and the monitoring of this prostatic disorder. $\beta$-Crosslap and prostate-specific antigen (PSA) were measured as a comparison. The serum samples of 210 patients were analyzed using a specific immunoassay. The patients were divided into seven groups according to the Whitmore Jewett Classification: 100 healthy subjects, 27 patients with benign prostate hyperplasia (BPH), 8 patients with prostate carcinoma level A, 17 patients with prostate carcinoma level $\mathrm{B}, 17$ patients with prostate carcinoma level C, 29 patients with prostate carcinoma level D, 12 patients were not assigned.

In patients with benign prostatic hyperplasia $\beta$-Crosslap showed a sensitivity of $51.85 \%$ and a specificity of $93 \%$. PSA showed a sensitivity of $62.96 \%$ and a specificity of $92 \%$ in the same cohort. Irrespective of the stage of prostate cancer $\beta$-Crosslap showed a sensitivity of $90.36 \%$ and a specificity of $100 \%$. PSA showed a sensitivity of $86.74 \%$ and a specificity of $100 \%$. If one refers to the different disease stages $\beta$-Crosslap showed a sensitivity of $75 \%$ at stage $A$, a sensitivity of $94.11 \%$ at stage $B$, a sensitivity of $88.23 \%$ at stage $C$ and a sensitivity of $96.55 \%$ at stage D. At all stages there was a specificity of $100 \%$. Elevated concentrations of this marker could always be detected. PSA showed a sensitivity of $62.5 \%$ at stage A, a sensitivity of $76.47 \%$ at stage $B$, a sensitivity of $88.23 \%$ at stage C and a sensitivity of $96.55 \%$ at stage $\mathrm{D}$. Elevated concentrations of this marker could always be detected. For the diagnosis of benign prostatic hyperplasia $\beta$-Crosslap does not provide any benefit due to its lower sensitivity. However, for the diagnosis of prostatic carcinoma one could profit from $\beta$-Crosslap as a prognostic marker especially at early stages of the disease.
\end{abstract}

\section{Introduction}

In Germany prostatic cancer is ranked first among all cancers in the male population showing an annual incidence of approximately 58,000 cases [1] which is higher than that of intestinal or lung cancer. Also in the United Stated prostate carcinoma was the most frequent cancer with about 218.890 new cases and about 27.050 deaths in 2007 [2]. The incidence increases with age and reaches a level of 1,000/100,000/year at the age of 80. Latent or occult carcinomas are not included in these figures. Therefore an even higher incidence can be assumed [3].

The "life time" risk to die from a clinically pronounced prostatic carcinoma is approximately $10 \%$ [3]. With an incidence of 65 to $75 \%$ the bone involvement, especially the metastatic spread into the axial skeleton is a major cause of the mortality and the morbidity [4]. Lower back pain may be symptoms of metastatic disease occur at an advanced and at the late stage [3].

On account of preventive medical check-ups and the currently available diagnostic measures the 5 year survival rate increased substantially in the past years [1].

Within this frame laboratory chemistry tests are available to assess the extent of the disease, the kind of the transformation and to monitor the course of the disease after a therapeutic intervention [3].

PSA can be detected in serum as prostate specific biomarker. This still leading tumor marker for the evaluation of disorders of the prostate gland does not show a sufficient sensitivity for the detection of prostate carcinomas.

Not only for this reason the search for new biomarkers with a higher specificity and sensitivity for the detection of a malignancy and for its staging and the assessment of the presence of metastases is of high importance [5].

In this context various products of metabolism play major roles which are formed during the bone remodeling and absorption driven by osteoblasts and bone absorbing osteoclasts, respectively. Furthermore, the associated regulation factors and ligands are of relevance. These biochemical markers have turned out to be a useful, noninvasive and comparatively inexpensive method for the assessment and monitoring of metastatic bone involvement [6].

$\beta$-Crosslap is a degradation product of bone metabolism. It is detectable in serum if bone metabolism is increased, e.g. in osteoporosis but also in patients with cancer where the primary tumor has impact on bone metabolism. This is enabled through certain mediators or tumor metastases [7-13].

Accordingly, subject of the present study was to measure sensitivity and specificity of the tumor marker $\beta$-Crosslap and to evaluate its clinical relevance for different disorders of the prostate gland.

Correspondence to: Lukas J. Becker, Departments of Laboratory Medicine Internal Medicine, Hospital of the Johann Wolfgang Goethe University, TheodorStern-Kai 7, D-60590, Frankfurt am Main, Germany, Tel: +49 69 63015024; Fax: +496963017201; E-mail: lukasjbecker@web.de

Key words: prostate carcinoma, $\beta$-Crosslap, PSA, tumor marker, bone metastases

Received: January 13, 2016; Accepted: January 25, 2016; Published: February 28,2016 


\section{Patients and methods}

This study comprised a cohort of 210 patients. Among them were 100 healthy subjects, 27 patients with benign prostatic hyperplasia, and 83 patients with prostatic carcinoma. The markers PSA and $\beta$-Crosslap were measured in the serum of patients. The measurement of the serum concentration was carried out through the immunologic in vitro test using the Elecsys 2010 measuring instrument by Roche Diagnostics Mannheim and the Johann Wolfgang Goethe University Frankfurt in Germany.

Patients with prostatic carcinoma were subsequently assigned to 4 groups according to the Whitmore Jewett

Classification to assess sensitivity and specificity of $\beta$-Crosslap at each of the disease stages.

\begin{tabular}{|l|l|c|}
\hline Group & $\begin{array}{c}\text { case number } \\
{[\mathbf{n}(\text { total })=210]}\end{array}$ \\
\hline $\mathbf{1}$ & healthy subjects & 100 \\
\hline $\mathbf{2}$ & $\begin{array}{l}\text { benign prostatic } \\
\text { hyperplasia }\end{array}$ & 27 \\
\hline $\mathbf{3}$ & $\begin{array}{l}\text { prostatic carcinoma } \\
\text { [total] }\end{array}$ & 83 \\
\cline { 2 - 3 } & Stage A & 8 \\
\hline & Stage B & 17 \\
\cline { 2 - 3 } & Stage C & 17 \\
\cline { 2 - 3 } & Stage D & 29 \\
\cline { 2 - 3 } & Not assigned & 12 \\
\hline
\end{tabular}

\section{Results}

\section{Diagnostic parameters of benign prostatic hyperplasia}

The results of diagnostic parameters for patients with benign prostatic hyperplasia are depicted in Table 1 and diagram 1 . It is obvious that $\beta$-Crosslap showing a specificity of $93 \%$ and a sensitivity of $51.85 \%$ is not more effective than PSA which shows a comparable specificity of $92 \%$ but a sensitivity of $62.96 \%$. The "Area under the ROC-Curve" also shows PSA to offer a better separation at 0.845185 than $\beta$-Crosslap at 0.745370 .

\section{Diagnostic parameters of prostatic carcinoma}

Specificity and sensitivity of tumor markers undergo considerable changes in prostatic carcinoma. If both tumor markers are compared with each other irrespective of the stage of the disease (Table 2 and diagram 2) $\beta$-Crosslap shows a specificity of $100 \%$ and a sensitivity of $90.36 \%$ at the optimal cut-off of $0.64 \mathrm{ng} / \mathrm{ml}$. At the same level of specificity PSA shows a sensitivity of only $86.74 \%$. Likewise, the Area under the ROC-

Curve shows an optimal separation for $\beta$-Crosslap at 0.960663 which is considerable lower for PSA at 0.880060 . If one looks at the tumor stages separately the properties of tumor markers deviate significantly in several stages from the overall picture shown in Table 2: At stage A $B$-Crosslap shows a specificity of $100 \%$ (Table 3 and diagram 3 ), however, a sensitivity of only $75 \%$. Likewise PSA shows at $100 \%$ specificity a sensitivity of only $62.5 \%$. If one takes also the AUC of the ROC-curve into consideration $B$-Crosslap is the more effective tumor marker at stage $\mathrm{A}$ of the disease.
Table 4 and diagram 4 show results of stage B prostatic carcinoma. At this stage $B$-Crosslap stands out as the more sensitive marker at an optimal cut-off value of 0.64 . Although PSA und $B$-Crosslap show a similar specificity of $100 \%$ the sensitivity of $94.11 \%$ versus $76.47 \%$ as compared to PSA at an optimal cut-off value of $6.0 \mathrm{ng} / \mathrm{ml}$ shows $B$-Crosslap to be the more effective marker. The area under the ROCCurve confirms the superior performance of $B$-Crosslap. At stage $C$ (Table 5 and diagram 5) both tumor markers match. At a specificity of $100 \%$ both show a similar sensitivity of $88.23 \%$. Only referring to the Area under the ROC-Curve a superior separation of $B$-Crosslap at 0.966716 stands out.

The optimal cut-off values being $1.48 \mathrm{ng} / \mathrm{ml}$ for $ß$-Crosslap and $14.7 \mathrm{ng} / \mathrm{ml}$ for PSA are relatively high as compared to the ones at subsequent tumor stages. As in stage $\mathrm{C}$ both tumor markers are on the same level at stage D. Both show a specificity of $100 \%$ and a sensitivity of $96.55 \%$.

\section{Discussion}

With respect to cause of death prostatic carcinoma is ranked third after deaths due to malignancies of lung and intestine representing approximately 11,000 deaths per year followed by pancreatic and gastric cancer [1]. The challenge of early detecting malignant changes of the prostate gland lies in the asymptomatic early stage [3].

PSA can be detected in serum as prostate specific biomarker. This still leading tumor marker for the evaluation of disorders of the prostate gland does not show a sufficient sensitivity for the detection of prostate carcinomas. Because PSA is susceptible to many disturbing factors it can be detected in serum in high concentrations just because of manipulations such as digital rectal examinations or other interventions. Non-malignant disorders of the prostate gland such as prostatitis result in increased levels also. Only in connection with further imaging diagnostics or the extraction of biopsies a reliable statement can be made $[3,14]$.

Actually Wymenga et al. found in their study that in $13 \%$ of patients with bone metastases the wrong diagnosis was made if PSA levels were the only diagnostic criteria [15].

Sophisticated measures such as the rectal ultrasound examination with or without spindle biopsy as well as X-ray, skeleton scintigraphy, MRT and MRT Lymphography to obtain tumor staging are indispensable $[2,14,15]$.

Furthermore Oremek et al. found that PSA has a low specificity for bone metastases. In their study, the specificity was 51.9\% [16]. Not only for this reason the search for new biomarkers with a higher specificity and sensitivity for the detection of a malignancy and for its staging and the assessment of the presence of metastases is of high importance. Several studies have shown that bone metastases were present in 83$90 \%$ of deceased patients with prostatic carcinoma and that these were causing the predominant symptoms during the course of the suffering [5]. In this regard the bone interaction of prostate disorders offers an opportunity to develop new diagnostic and therapeutic procedures for screening and follow-up programs [5].

Objective of this study was to assess the validity of $\beta$-Crosslap concerning prostate carcinoma and to evaluate its benefit in the early detection, the diagnosis and the monitoring of this prostatic disorder.

\section{Validity of $\beta$-Crosslap}

The sample material was obtained from patients with prostate 
Table 1. Diagnostic parameters of BPH

\begin{tabular}{|l|l|l}
\hline & PSA & B-Crosslap \\
\hline \hline Cut-off & & \\
\hline sensitivity & $3.7 \mathrm{ng} / \mathrm{ml}$ & $0.415 \mathrm{ng} / \mathrm{ml}$ \\
\hline specificity & $62.96 \%$ & $51.85 \%$ \\
\hline \hline area under the ROC-Curve & $92 \%$ & $93 \%$ \\
\hline median & 0.845185 & 0.745370 \\
\hline standard deviation & 4.1 & 0.42 \\
\hline
\end{tabular}

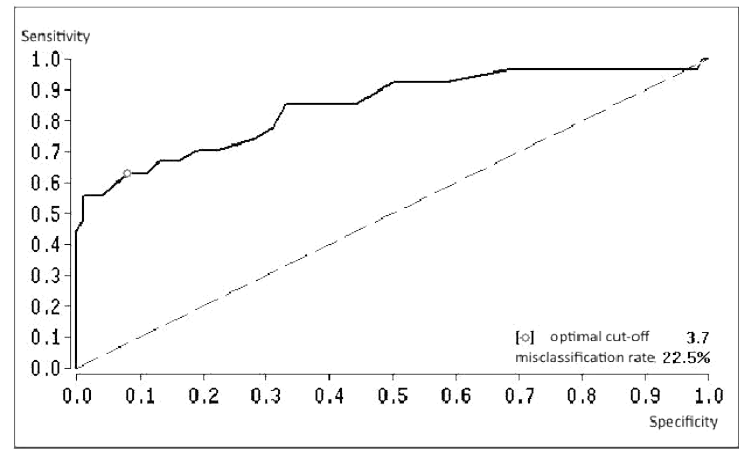

Diagram 1a: ROC-Analysis PSA

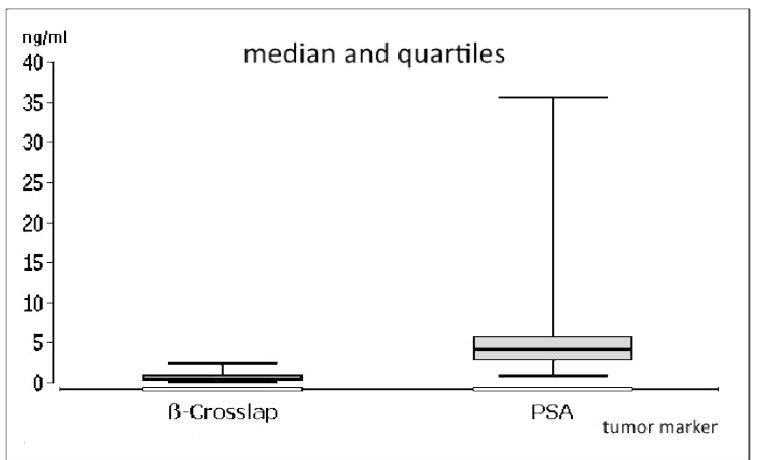

Diagram 1c: Box-Plots

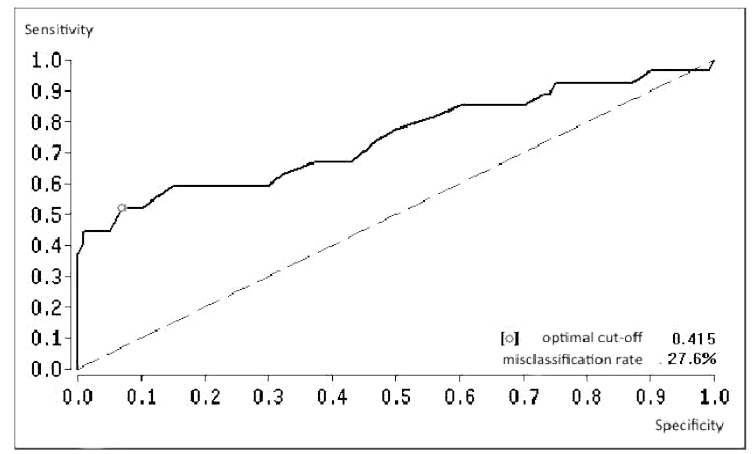

Diagram 1b: ROC-Analysis $\beta$-Crosslap

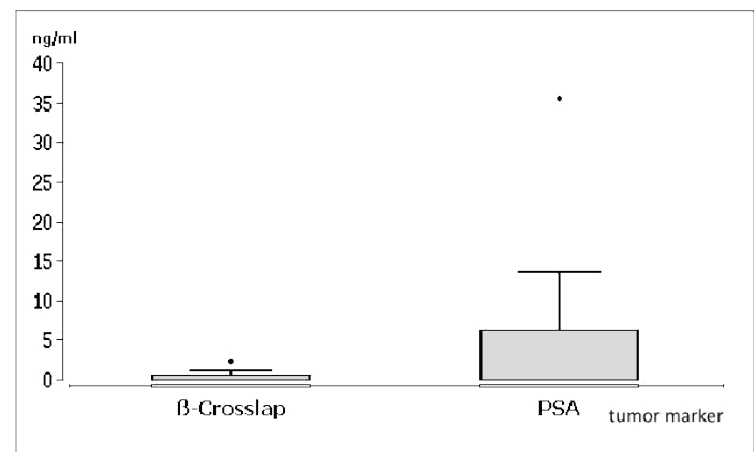

Diagram 1d: Bar-Plots Average values and standard deviations

Table 1 shows measurement results for PSA and $\beta$-Crosslap in patients with benign prostatic hyperplasia.

Like PSA (62.96\%) $\beta$-Crosslap shows a low sensitivity (51.85\%). PSA shows a specificity of $92 \%$ and $\beta$-Crosslap one of 93\%. The standard deviation is high for PSA (7.3611). For $\beta$-Crosslap the standard deviation is 0.6089

Sensitivity and specificity as well as the cut-off value are shown in diagram 1a and 1b. Average values with the corresponding standard deviations and the quartiles are shown in diagram 1c and 1d 
Table 2. Diagnostic parameters for patients with prostatic carcinoma (in total).

\begin{tabular}{|l|l|l|}
\hline & PSA & B-Crosslap \\
\hline Cut-off & 4.45 & 0.64 \\
\hline sensitivity & $86.74 \%$ & $90.36 \%$ \\
\hline specificity & $100 \%$ & $100 \%$ \\
\hline area under the ROC-Curve & 0.880060 & 0.960663 \\
\hline median & 38.2 & 7.4 \\
\hline standard deviation & 933.2039 & 29.2910 \\
\hline
\end{tabular}

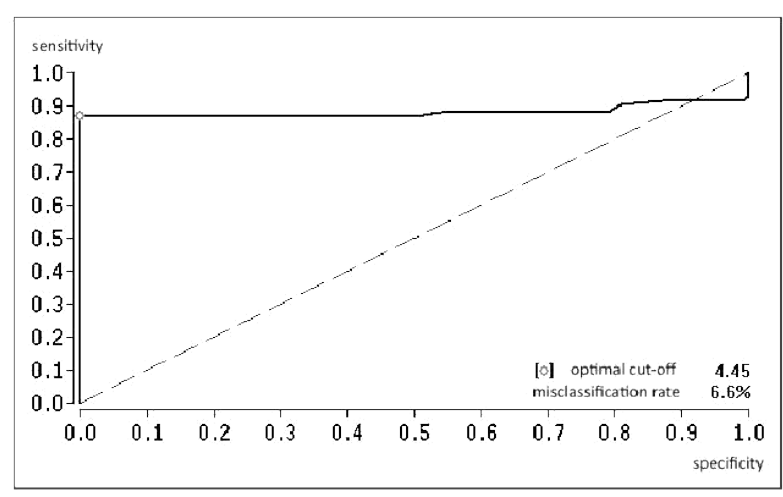

Diagram 2a: ROC-Analysis PSA

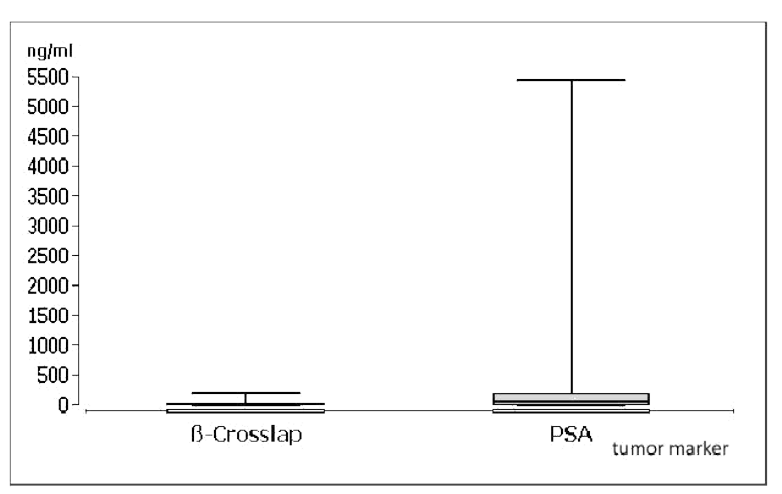

Diagram 2c: Box-Plot median and quartiles

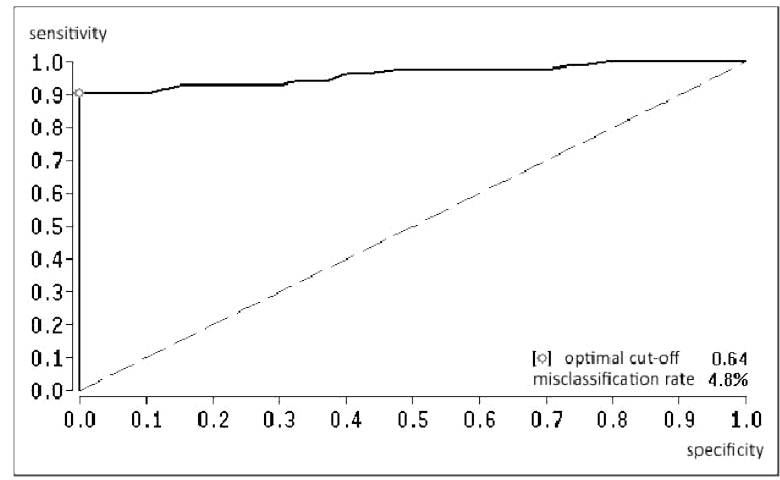

Diagram 2b: ROC-Analysis $\beta$-Crosslap

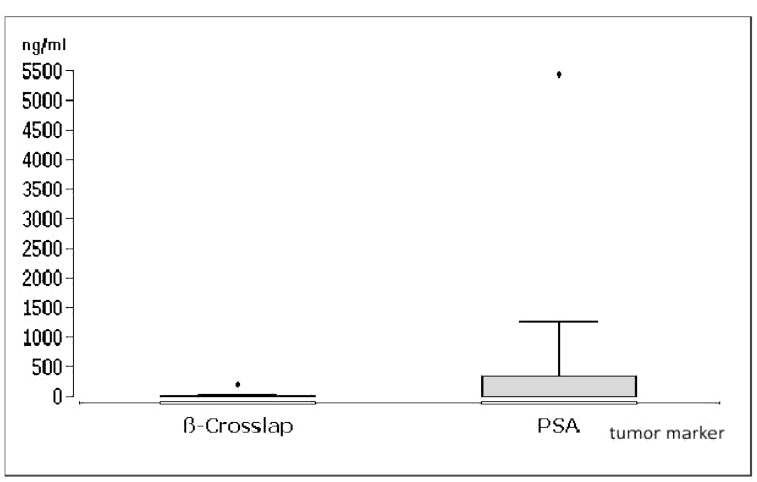

Diagram 2d: Bar-Plots Average values and standard deviations

In Table 2 the diagnostic parameters of PSA and $\beta$-Crosslap in prostatic carcinoma are depicted irrespective of the stage of the disease. The sensitivity of $\beta$-Crosslap of $90.36 \%$ is higher than that of PSA (86.74\%) at a specificity of $100 \%$. The standard deviation of PSA is very high at 933.2039. For $\beta$-Crosslap it is 29.2910 . Sensitivity and specificity as well as the Cut-off-value are depicted in diagram 2a and 2b. Average values with corresponding standard deviations and quartiles are depicted in diagram $\mathbf{2 c}$ and $\mathbf{2 d}$. 


\begin{tabular}{|l|l|l|}
\hline & PSA & B-Crosslap \\
\hline Cut-off & $4.9 \mathrm{ng} / \mathrm{ml}$ & $0.69 \mathrm{ng} / \mathrm{ml}$ \\
\hline sensitivity & $62.5 \%$ & $75 \%$ \\
\hline specificity & $100 \%$ & $100 \%$ \\
\hline Area under the ROC-Curve & 0.675000 & 0.871875 \\
\hline Median & 12.9 & 3.3 \\
\hline standard deviation & 16.6996 & 2.8957 \\
\hline
\end{tabular}

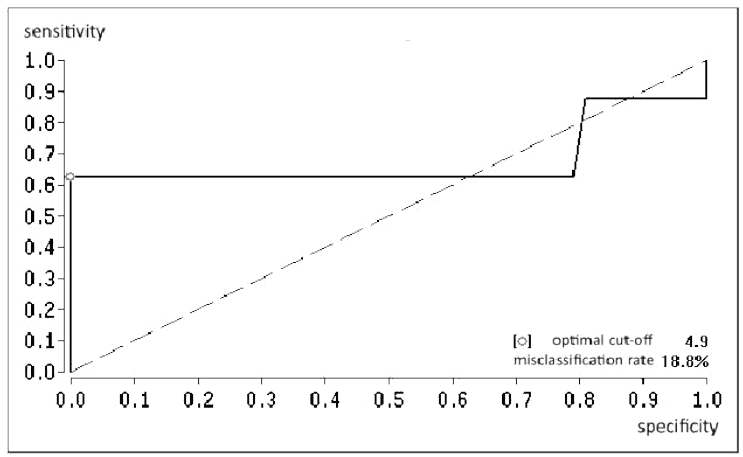

Diagram3a: ROC-Analyse PSA

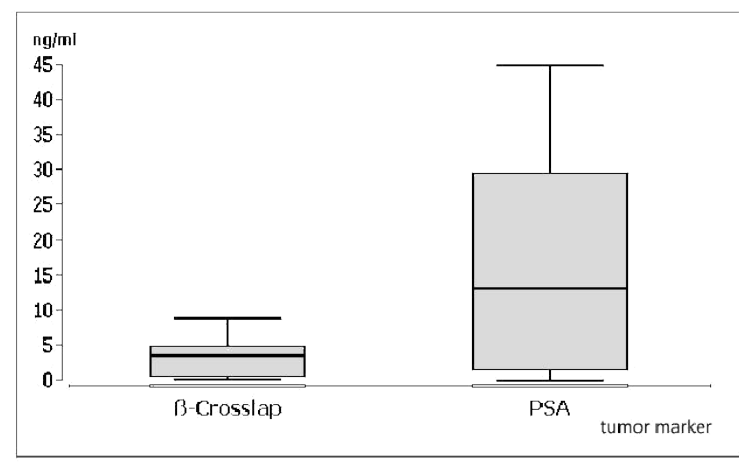

Diagram 3c: Box-Plots median and quartiles

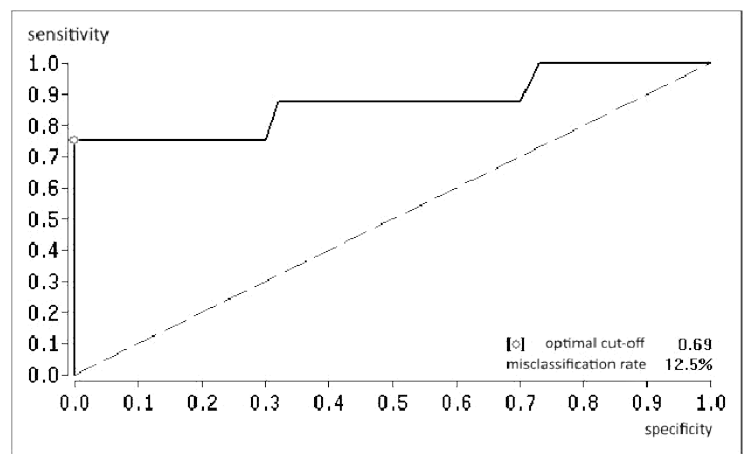

Diagram 3b: ROC-Analyse $\beta$-Crosslap

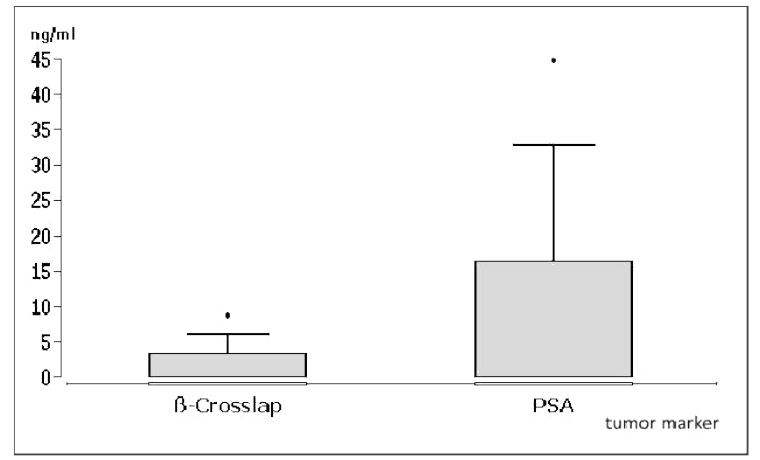

Diagram 3d: Bar-Plots Average values and standard deviations

Table 3 shows diagnostic parameters for PSA and $\beta$-Crosslap at stage A prostatic carcinoma. he sensitivity of $\beta$ Crosslap of $75 \%$ is higher than that of PSA $(62.5 \%)$ at the same specificity of $100 \%$. The standard deviation of PSA is 16.6996 . For $\beta$-Crosslap it is 2.8957 . Sensitivity and specificity as well as the Cut-off value are depicted in diagram 3a and 3b. Average values with corresponding standard deviations and quartiles are shown in diagram 3c and 3d. 
Table 4. Diagnostic parameters for patients with stage B prostatic carcinoma.

\begin{tabular}{|l|l|l|}
\hline & PSA & B-Crosslap \\
\hline Cut-off & $6.0 \mathrm{ng} / \mathrm{ml}$ & $0.64 \mathrm{ng} / \mathrm{ml}$ \\
\hline sensitivity & $76.47 \%$ & $94.11 \%$ \\
\hline specificity & $100 \%$ & $100 \%$ \\
\hline Area under the ROC-Curve & 0.793235 & 0.977353 \\
\hline Median & 10.5 & 2.4 \\
\hline Standardabweichung & 10.5314 & 2.4589 \\
\hline
\end{tabular}

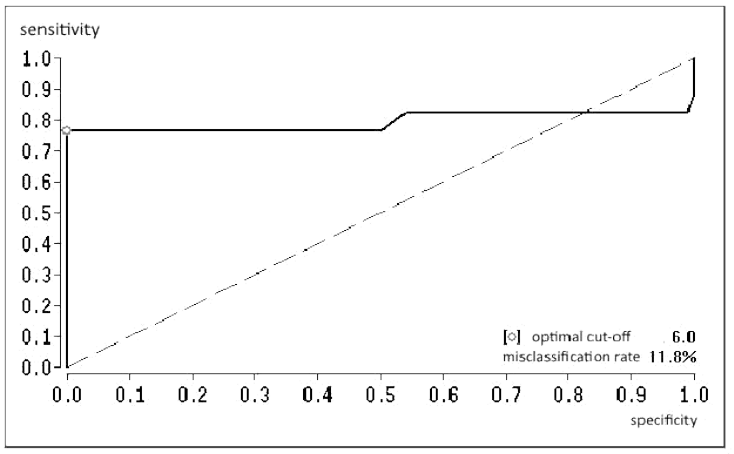

Diagram 4a: ROC-Analyse PSA

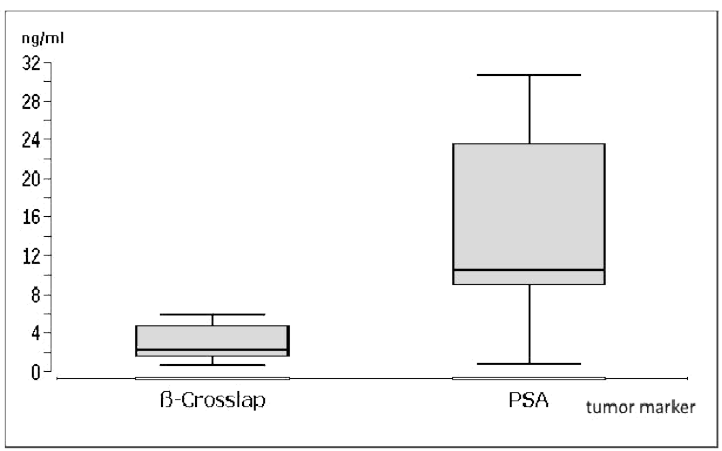

Diagram 4c: Box-Plots median and quartiles

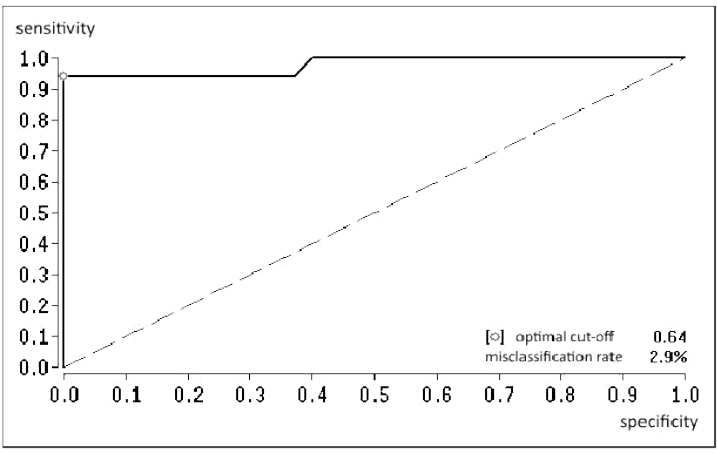

Diagram 4b: ROC-Analyse $\beta$-Crosslap

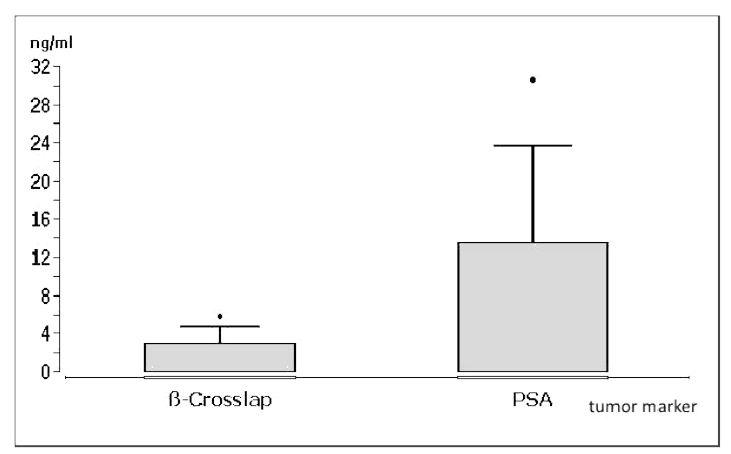

Diagram 4d: Bar-Plots Average values and standard deviations

Table 4 shows diagnostic parameters for PSA and $\beta$-Crosslap in stage B prostatic carcinoma. At the same specificity of $100 \% \beta$-Crosslap shows a sensitivity of $94.11 \%$ which is higher than that of PSA (76.4\%). The standard deviation of PSA is 10.5314 . For $\beta$-Crosslap it is 2.4589 . Sensitivity and specificity as well as the Cut off value are depicted in diagram $4 \mathbf{a}$ and $\mathbf{4 b}$. Average values with corresponding standard deviations are shown in diagram $4 c$ and $4 d$. 


\begin{tabular}{|l|l|l|}
\hline & PSA & 及-Crosslap \\
\hline Cut-off & $14.7 \mathrm{ng} / \mathrm{ml}$ & $1.48 \mathrm{ng} / \mathrm{ml}$ \\
\hline sensitivity & $88.23 \%$ & $88.23 \%$ \\
\hline specificity & $100 \%$ & $100 \%$ \\
\hline Area under the ROC-Curve & 0.891765 & 0.966176 \\
\hline Median & 80.4 & 11.2 \\
\hline Standardabweichung & 342.0667 & 23.6475 \\
\hline
\end{tabular}
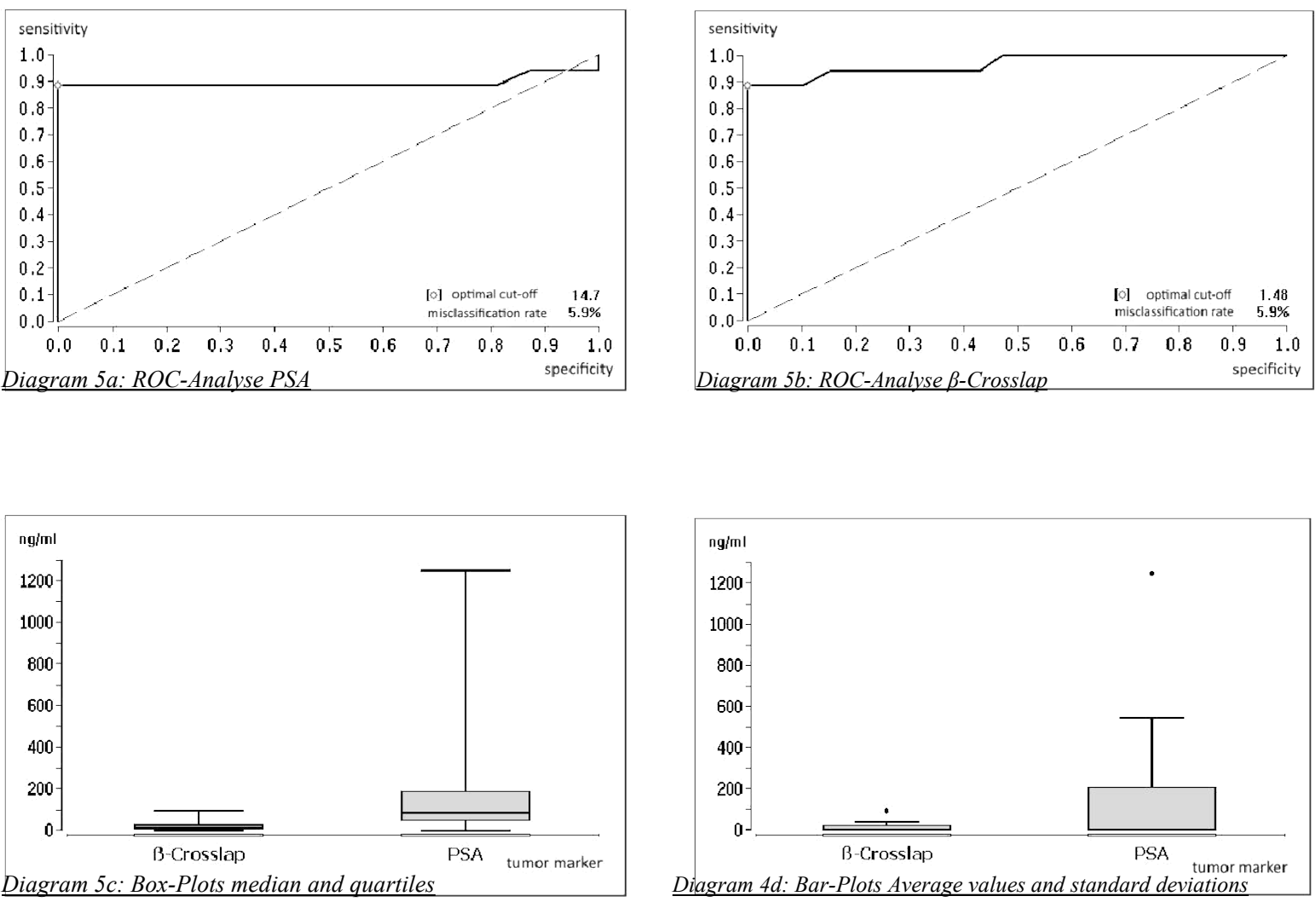

Table 5 shows diagnostic parameters for PSA and $\beta$-Crosslap in stage $C$ prostatic carcinoma. $\beta$-Crosslap and PSA both show a sensitivity of $88.23 \%$ and a specificity of $100 \%$. The standard deviation of PSA is 342.0667 . For $\beta$-Crosslap it is 23.6475 . Sensitivity and specificity as well as the Cut-off value are depicted in diagram 5a and 5b. Average values with corresponding standard deviations and quartiles are shown in diagram 5c and 5d. 
Table 6. Diagnostic parameters for patients with stage D prostatic carcinoma.

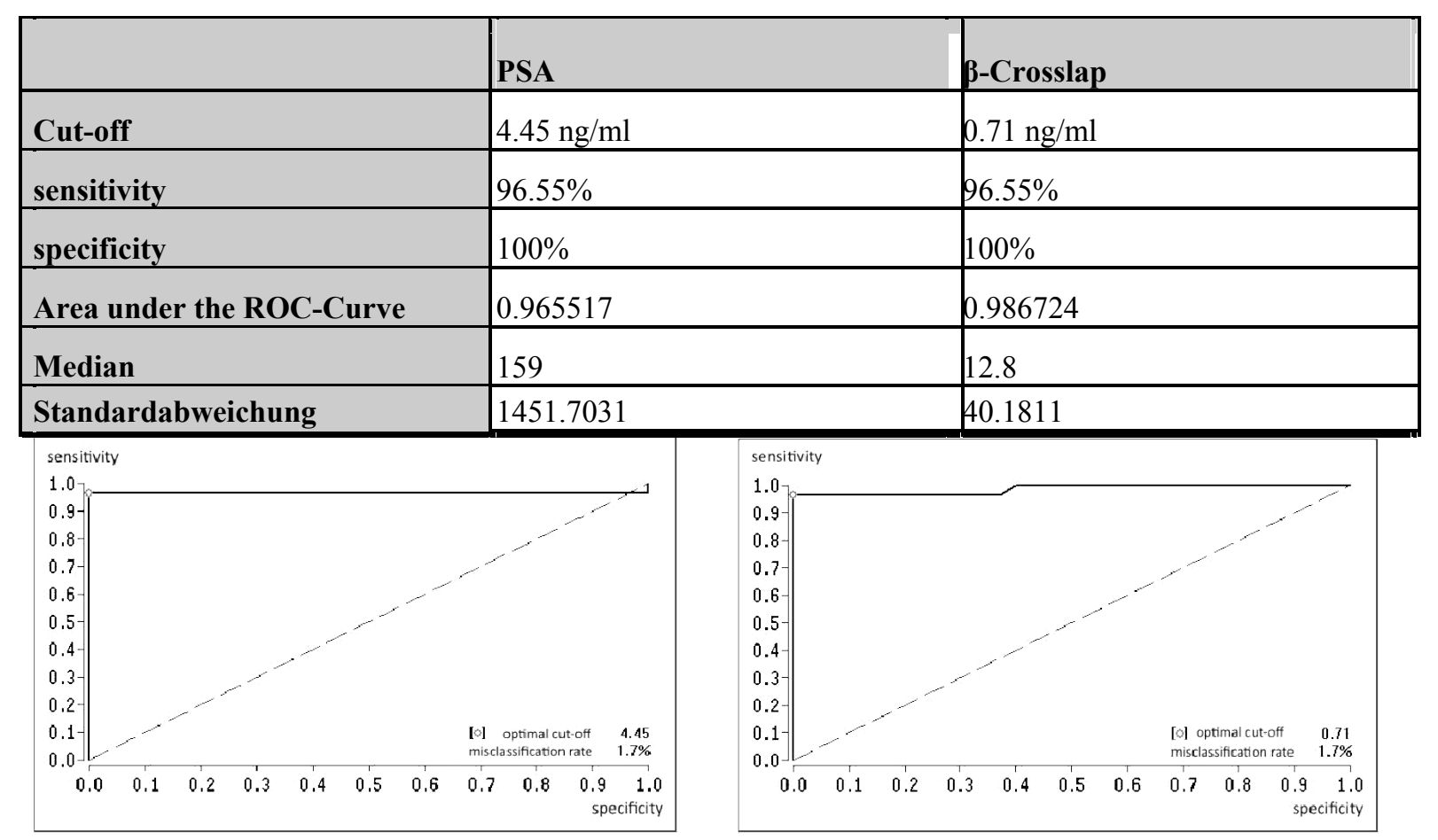

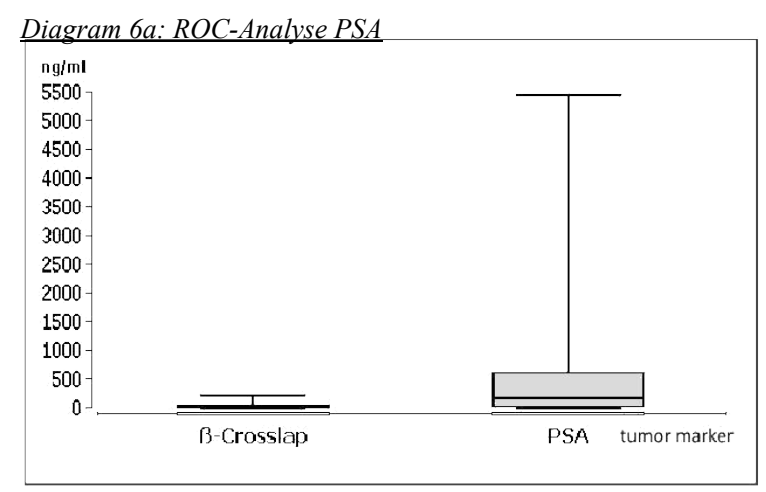

Diagram 6c: Box-Plots median and quartiles

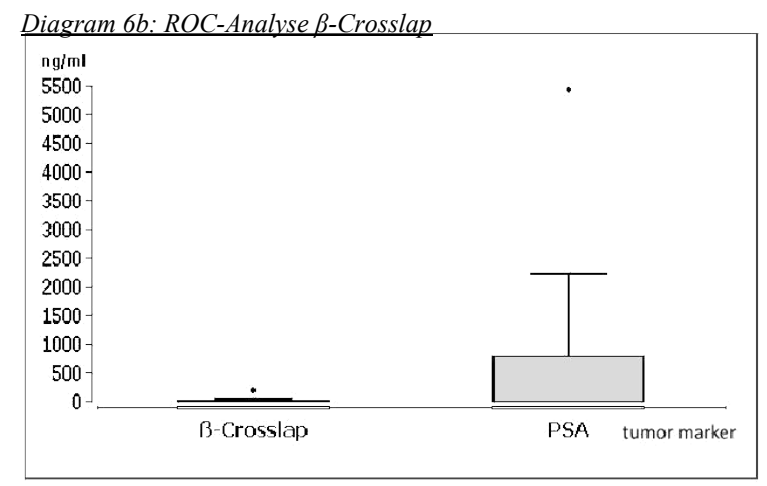

Diagram 6d: Bar-Plots Average values and standard deviations

Table 6 shows diagnostic parameters for PSA and $\beta$-Crosslap in stage D prostatic carcinoma. $\beta$-Crosslap and PSA show a sensitivity of $96.55 \%$ and a specificity of $100 \%$. The standard deviation of PSA is 1451.7031 . For

$\beta$-Crosslap it is 40.1811 . Sensitivity and specificity as well as the Cut-off value are depicted in diagram 6a and 6b. Average values with corresponding standard deviations and quartiles are shown in diagram $6 \mathbf{c}$ and $6 \mathbf{d}$. 
carcinoma, from patients with benign prostatic hyperplasia as well as from healthy subjects.

This material was used to measure the tumor marker PSA and $\beta$-Crosslap. Values obtained from both markers in various prostatic disorders were correlated and assessed which revealed significant differences of the validity within the respective disorder categories.

\section{Validity of $\beta$-Crosslap in patients with benign prostatic hyperplasia}

In the group of patients with benign prostatic hyperplasia the tumor markers PSA and $\beta$-Crosslap were measured in 27 patients. In this process $\beta$-Crosslap showed a slightly higher specificity of $93 \%$ than PSA of $92 \%$. Its sensitivity of $51.85 \%$ was significantly lower than that of PSA of $62.96 \%$.

Table 1 shows the average value of PSA to be slightly increased at $4.1 \mathrm{ng} / \mathrm{ml}$. In six patients a concomitant urinary tract infection was documented and in one patient a chronic unspecified inflammation of the prostate gland. One further patient was under long term treatment with a bladder catheter. These secondary diagnoses and this concomitant treatment may explain an additional increase of the PSA value and the increased average value. All of this may account for the high standard deviation of 7.3611 and the low sensitivity of $62.96 \%$.

In three additional patients secondary diagnoses of malignant diseases were present. One patient was suffering from lung cancer, one patient from cancer of the esophagus and the third one from colon cancer. Two more patients were suffering from tetra spastic and from Parkinson's disease. At the time of conducting this study staging of malignant diseases was not available. In one looks at the diagnostic parameters in Table 1 the average value of $\beta$-Crosslap is increased at 0.42 .

In the case of benign prostatic hyperplasia it can be assumed bone tissue not to be affected since the disease is confined to the increase of prostatic tissue. Since some of the patients were suffering from malignant processes the increased value of $\beta$-Crosslap may be due to affection of bone tissue through these secondary diagnoses. W. Ebert and colleagues were able to show a connection between lung cancer and the increase of CTX values [17]. They point out, however, that this connection if given only in case of metastasis. At stages without metastasis no significant increase was found [17].
Oremek and colleagues [18,19] illustrate in their studies the association of metastasized tumors with the increase of markers of bone formation. Primarily in metastasized colon and liver carcinoma as well as in advanced breast cancer increased $\beta$-Crosslap values were detectable in the blood of affected patients. Due to the abnormally high values which correlated with tumor stages Oremek and colleagues suggested to use $\beta$-Crosslap as a new marker for the assessment and the treatment of patients suffering from stages of these malignant diseases in which metastases are present $[18,19]$.

\section{Validity of $\beta$-Crosslap in patients with prostatic carcinoma}

Patients with prostatic carcinoma were assigned to stages of the disease according to the Whitmore-Jewett Classification. Accordingly, 8 subjects were at stage A, 17 at stage B and 17 at stage C. 29 subjects were assigned to stage $\mathrm{D}$. In 7 subjects concomitant diseases of the kidney or the urinary tract were present like renal insufficiency in 4 cases, one case of interstitial cystitis, one case of hydronephrosis and one case of renal pelvic stone.

One patient was suffering from bronchial asthma whereas another one was diagnosed with pulmonary emphysema. 5 patients were suffering from Diabetes mellitus. Two subjects were diagnosed with ulcerative colitis. One subject presented with hepatocellular carcinoma as secondary malignancy.

If one looks at the measurement results shown in Diagram 7 it becomes apparent that $B$-Crosslap values are higher than those of healthy subjects. In addition, they correlate with tumor progression: The median of the measured results lies at $3.3 \mathrm{ng} / \mathrm{ml}$ at stage $\mathrm{A}$, at 2.4 $\mathrm{ng} / \mathrm{ml}$ at stage $\mathrm{B}$, at $11.2 \mathrm{ng}$ at stage $C$ reaching $12.8 \mathrm{ng} / \mathrm{ml}$ at stage D. Likewise, PSA shows a stage dependent increase of measurement results in accordance with tumor progression.

However, a significant difference between both markers lies in their standard deviations. Whereas this assumes great dimensions in case of PSA it is relatively minor in case of $\beta$-Crosslap. At stage A a standard deviation of

16.6996 for PSA and of 2.8957 for $\beta$-Crosslap, respectively were calculated. The higher the stage the higher the associated standard deviation: At stage D the standard deviation of 1451.7031 for PSA and of 40.1811 for $\beta$-Crosslap illustrate this.

Diagram 7. Overview of $\beta$-Crosslap values. Diagrams 7a and $\mathbf{7 b}$ show the average values, the standard deviations as well as the quartiles of tumor stages in a direct comparison with the changes they undergo at increasing tumor stages.

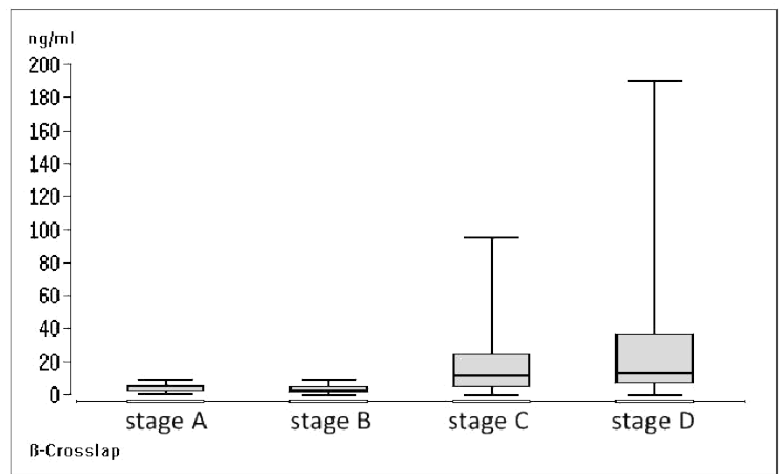

Diagram 7a: Box-Plots median and quartiles

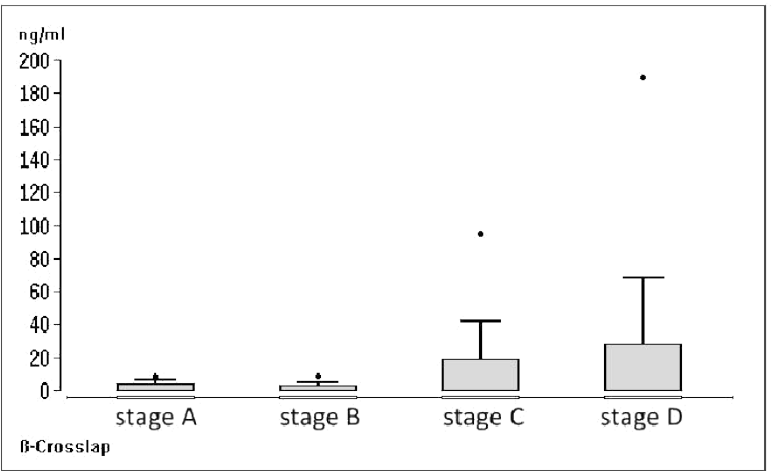

Diagram 7b: Bar-Plots Average values and standard deviations 
If one compares PSA with B-Crosslap at each of the tumor stages it becomes obvious that $B$-Crosslap offers a higher validity especially at the stages A and B. At stage A the sensitivity of $75 \%$ of $B$-Crosslap is superior to the one of PSA of $62.5 \%$ at the same level of specificity. Also at stage B ß-Crosslap suggests to have a higher sensitivity of $94.11 \%$ whereas PSA at that stage shows a sensitivity of $76.47 \%$ only.

At stages $\mathrm{C}$ and $\mathrm{D}$ both markers show the same sensitivity and specificity. However, if one takes into consideration the separation (AUC) as well as the standard deviation it becomes obvious ß-Crosslap to be less susceptible to interfering factors.

\section{Conclusion}

If one refers to the results of each stage of prostate carcinoma separately $\beta$-Crosslap shows to be more sensitive than PSA. Especially at the early stages $\beta$-Crosslap proved to offer a significantly higher sensitivity in the diagnostic of metastases. At later stages of the disease the values of both markers are similar. However, the susceptibility of $\beta$-Crosslap to influencing factors is lower which also offers an advantage over PSA.

For the diagnosis of benign prostatic hyperplasia $\beta$-Crosslap does not provide any benefit due to its lower sensitivity. However, for the diagnosis of prostatic carcinoma one could profit from $\beta$-Crosslap as a prognostic marker especially at early stages of the disease.

\section{References}

1. Robert Koch Institut (RKI) (2008) Gesellschaft der epidemiologischen Krebsregister in Deutschland (GEKID). Krebs in Deutschland 2003-2004. Häufigkeiten und Trends.6th ed. Berlin: RKI.

2. American Cancer Society (2007) . Cancer Facts and Figures American Cancer Society, Atlanta.

3. Haag P, Hanhart N, Müller M (2009) Gynäkologie und Urologie für Studium und Praxis, 5. Auflage, 2010/2011 Medizinische Verlags und Informationsdienste. Breisach( Rh.) $347-356$.

4. Coleman RE (1997) Skeletal complications of malignancy. Cancer 80: 1588-1594. [Crossref]

5. Mizutani K, Sud S, Pienta KJ (2009) Prostate cancer promotes CD11b positive cells to differentiate into osteoclasts. J Cell Biochem 106: 563-9. [Crossref]

6. Seibel MJ, Woitge HW (1999) Basic principles and clinical applications of biochemical markers of bone metabolism: biochemical and technical aspects. J Clin Densitom 2: 299-321. [Crossref]
7. Christgau S, Cloos PA (2003) Current and future applications of bone turnover markers Clin Lab 49: 439-446. [Crossref]

8. Delmas PD, Eastell R, Garnero P, Seibel MJ, Stepan J, et al. (2000) The use of biochemical markers of bone turnover in osteoporosis. Committee of Scientific Advisors of the International Osteoporosis Foundation. Osteoporos Int 11 Suppl 6: S2-17. [Crossref]

9. Bonde M, Qvist P, Fledelius C, Riis BJ, Christiansen C (1994) Immunoassay for quantifying type I collagen degradation products in urine evaluated. Clin Chem 40: 2022-2025. [Crossref]

10. Fledelius C, Johnsen AH, Cloos PA, Bonde M, Qvist P (1997) Characterization of urinary degradation products derived from type I collagen. Identification of a betaisomerized Asp-Gly sequence within the C-terminal telopeptide (alpha1) region. J Biol Chem 272: 9755-9763. [Crossref]

11. Bonde M, Qvist P, Fledelius C, Riis BJ, Christiansen C (1995) Applications of an Enzyme Immunoassay for a New Marker of Bone Resorption (CrossLaps): Followup on Hormone Replacement Therapy and Osteoporosis Risk Assessment. J Clin Endocrinol Metab 80: 864-868. [Crossref]

12. Ravn P, Clemmesen B, Riis BJ, Christiansen C (1996) The Effect on Bone Mass and Bone Markers of Different Doses of Ibandronate: A New Bisphosphonate for Prevention and Treatment of Postmenopausal Osteoporosis. A 1-year, Randomized, Double-Blind, Placebo-Controlled Dose-Finding Study. Bone 19: 527-533. [Crossref]

13. Rosenquist C, Fledelius C, Christgau S, Pedersen BJ, Bonde M, et al. (1998) Serum CrossLaps One Step ELISA. First application of monoclonal antibodies for measurement in serum of bone-related degradation products from C-terminal telopeptides of type I collagen. Clin Chem 44: 2281-2289. [Crossref]

14. Jürgen Sökeland, Herbert Rübben (2008) Taschenlehrbuch Urologie 14. Auflage Georg Thieme Verlag 1955, 2008 , Stuttgart, New York; 27-28, 312-342.

15. Wymenga LF, Boomsma JH, Groenier K, Piers DA, Mensink HJ (2001) Routine bone scans in patients with prostate cancer related to serum prostate-specific antigen and alkaline phosphatase. BJU Int 88: 226-230. [Crossref]

16. Klepzig M, Jonas D, Oremek GM (2009) Procollagen type 1 amino-terminal propeptide: a marker for bone metastases in prostate carcinoma. Anticancer Res 29: 671-673. [Crossref]

17. Ebert W, Muley T, Herb KP, Schmidt-Gayk H (2004) Comparison of bone scintigraphy with bone markers in the diagnosis of bone metastasis in lung carcinoma patients. Anticancer Res 24: 3193-3201. [Crossref]

18. Oremek GM, Weis A, Sapoutzis N, Sauer-Eppel H (2003) Diagnostic value of bone and tumour markers in patients with malignant diseases. Anticancer Res 23: 987-990. [Crossref]

19. Oremek GM, Sapoutzis N, Lorenz M (2003) Phospholipids, tumour marker and betaCrossLaps in diagnosis of gastric carcinoma. Anticancer Res 23: 859-863. [Crossref]

Copyright: (C2016 Becker LJ. This is an open-access article distributed under the terms of the Creative Commons Attribution License, which permits unrestricted use, distribution, and reproduction in any medium, provided the original author and source are credited. 